\title{
MPC-Based Arctan Droop Control Strategy of the Parallel Inverter System in an Islanded AC Microgrid
}

\author{
J. D. D. Iyakaremye $\mathbb{D}^{1},{ }^{1}$ G. N. Nyakoe ${ }^{D},^{2}$ and C. W. Wekesa ${ }^{1}{ }^{1}$ \\ ${ }^{1}$ Department of Electrical Engineering, Pan African University Institute for Basic Sciences, \\ Technology, and Innovation (PAUSTI), P.O. Box 62000-00200, City Square, Nairobi, Kenya \\ ${ }^{2}$ Department of Electrical Engineering, Jomo Kenyatta University of Agriculture and Technology (JKUAT), \\ P.O. Box 62000-00200, City Square, Nairobi, Kenya
}

Correspondence should be addressed to J. D. D. Iyakaremye; iyakjdd@gmail.com

Received 23 August 2020; Revised 4 February 2021; Accepted 28 February 2021; Published 11 March 2021

Academic Editor: Kamran Iqbal

Copyright ( $\odot 2021$ J. D. D. Iyakaremye et al. This is an open access article distributed under the Creative Commons Attribution License, which permits unrestricted use, distribution, and reproduction in any medium, provided the original work is properly cited.

\begin{abstract}
Distributed generations (DG) are one of the upcoming technologies recently used by many electric utilities in all corners of the world. Most of those DG form the microgrid (MG) to serve local loads and can be connected to the grid. This DG's technology is enabled by utilizing renewable energy sources (REs) that are ecofriendly; however, these REs are intermittent by their nature, so controlling a power electronic device interfaced with them to be connected to the grid is another challenge. Many researchers have worked on the inverters' control in MG. This study also elaborates on the control strategy for inverters adapted to REs for proper control of voltage and frequency used in an islanded microgrid. The study proposes a hybrid control strategy made of the virtual impedance droop control with arctan function and model predictive control. Extensive simulations have been carried out to validate the proposed control strategy's effectiveness in terms of rapid transient response and stabilization of voltage, frequency, and power equitability among the microsources in the islanded microgrid.
\end{abstract}

\section{Introduction}

Distributed generations (DG) are seen as alternative solutions to combat pollution and to meet the growing demand for electricity in recent years [1]. A network approach that views the generator and related loads as a module or "microgrid" is a better way of understanding the distributed generation evolving promise [2]. The reduction in the conventional economy of scale in central power generation facilities gives way to a lower, more dispersed generation. A broad range of prime mover technologies, such as internal combustion engines (IC), gas turbines, microturbines, photovoltaic fuel cells, and wind turbines, is used in distributed generation. The bulk of emerging developments such as microturbines, photovoltaics, fuel cells, and wind energy sources are interfaced to the grid with an inverter. These new technologies have lower costs and are theoretically less expensive than conventional systems [2].

Power electronics converters are the most critical item for any power conditioning process used in a microgrid to satisfy various sources/loads. Therefore, either a single microsource or parallel microsource is very critical for optimizing their operation. Many control techniques have been proposed for the three-phase voltage source inverters (VSI) [3]. The most widely used are droop control techniques, deadbeat control, predictive control, and cascaded linear control [4-6].

Predictive control and droop control occupy a wide variety of controls that have been established to control power converters. To address existing challenges such as nonlinearity, slow response, unequal power sharing, and complexity of the control design, the arctan function droopbased model predictive control technique is used in this 
study to control the voltage, frequency, and power sharing of the voltage source inverter in a microgrid.

Some researchers worked on the droop control in conjunction with model predictive control (MPC) to control the microgrid. Babqi and Etemadi [7] used MPC as a primary control and conventional droop control as a secondary control; both were used to regulate the output voltage and frequency of each DG-based VSI in an autonomous microgrid. Finite control set MPC has been used by Dragicevic [8] as a control method based on precise tracking of derivative's reference voltage to regulate the voltage at the shared bus, and a simple traditional droop controller was used for power sharing among the VSIs. Chen et al. [9] have proposed an MPC-based power sharing control strategy and the current estimator in the capacitor to minimize the additional current sensor; in this method, a traditional reverse droop controller and virtual resistive concept were used to ensure power sharing.

Bouzid et al. [10] proposed the new control method for stable current distribution applied to parallel microgrid inverters systems, based on a decoupled trigonometric saturation regulator (DTS). Introduced with the latest DTS control, energy distribution has been improved with more excellent stability and an efficient energy efficiency level. Active and reactive power dynamic decoupling in the presence of various impedances, also this method, ensures voltage and frequency stabilities; however, the technique utilized the secondary control loop based on proportional integral (PI) for voltage and frequency restoration, which operates at the slow dynamic response and increases the complexity of control strategy design. The new concept utilizing the arctan function for the power frequency droop profile has been introduced in $[11,12]$. Using this arctan function improves the small stability of the two inverters' microgrid signal, offers a natural frequency limit, and is flexible in its application; however, this discussed method presents poor voltage regulation.

Zhongwen et al. [13] proposed a fully distributed approach to hierarchical management. It combines secondary and tertiary monitoring into a single control point. Detailed switches' designs are used instead of standard versions for paralleled VSIs connected to the grid; the method used droop control and virtual impedance for voltage, frequency control, and adequate power sharing. However, the introduction of virtual impedance may result in the voltage drop in the system; thus, a proper selection of virtual impedance value must be considered.

Finite control set MPC (FCS-MPC) is based on a radically different concept, as opposed to linear control $[14,15]$. Instead of developing loops for each controlled variable individually and then cascading them together, FCS-MPC uses the VSI mathematical model to forecast its future behavior and then evaluate the power converter's optimum switching status by the specified objective function. Cortés et al. [16] proposed a standard FCS-MPC scheme for a threephase inverter with an output LC filter. The proposed method achieved effective voltage control with linear loads and also with nonlinear loads.

Zheng et al. [17] have recommended that the hardware budget be reduced and system efficiency be enhanced by decreasing existing MPC sensors. FCS-MPC provides the advantages of robustness, outstanding transient properties, and incorporation of nonlinear variables, constraints, and external control objectives; these have proved to be an essential candidate for VSIs regulation $[18,19]$. The work on MPC-dependent droop control in the coordinated control of parallel inverters in a microgrid is still immature to the best of the authors' knowledge. A lot can be performed to increase such control strategy's capability in hardware simplification, robustness, and transient response. The use of a control method dependent on MPC requires a heavier computational burden; therefore, using a powerful processor is required; this may result in a different budget [20]. With the recent development of modern processors, this can be managed.

The conventional droop control strategies dependent on linear droop function have a common drawback where the corresponding output range scaling factor does not affect system dynamics and steady state. Therefore, there is a possibility that the system frequency can largely deviate from the nominal value if the same is determined through the droop control [21].

This study introduces a new control method combining the arctan virtual impedance droop control strategy for primary voltage and frequency control and power sharing issues, and FCS-MPC strategy is used for tracking the reference voltage. An advanced FCS-MPC technique for controlling the voltage and choosing appropriate control behavior in the next phase is implemented. With hierarchical linear regulation, the proposed approach can achieve comparable steady state efficiency while enhancing the transitional response. The control design expenditure is reduced concerning current cascaded linear control-based systems as there is no need for voltage and current loops in the control strategy.

The study's sections are arranged as follows: the AC microgrid structure is defined in Section 2. Section 3 sets out the existing control theory for VSI. Section 4 describes the proposed control algorithm and its use in VSIs based on an alternating current (AC) microgrid. The results and discussion are presented in Section 5, and finally, Section 6 ends with a conclusion.

\section{Description of the System}

Figure 1 displays a VSI-based MG. It is composed of DG units that are parallel linked via the VSI. Since the DGs are usually connected to the electricity storage device to ensure a stable power supply, direct current sources are considered the inverters' inputs [22]. The load is shared through the same AC bus known as the common coupling point. The MG can be linked to the primary grid or run in the autonomous mode.

A control strategy has been designed to control the paralleled VSIs better to assure the MG's proper control. Therefore, the MPC-based arctan droop controller with virtual impedance is used to ensure voltage and frequency stabilization; moreover, the power equitability among the microsources is assured. Each part of this hybrid control strategy will provide adequate energy management and the 


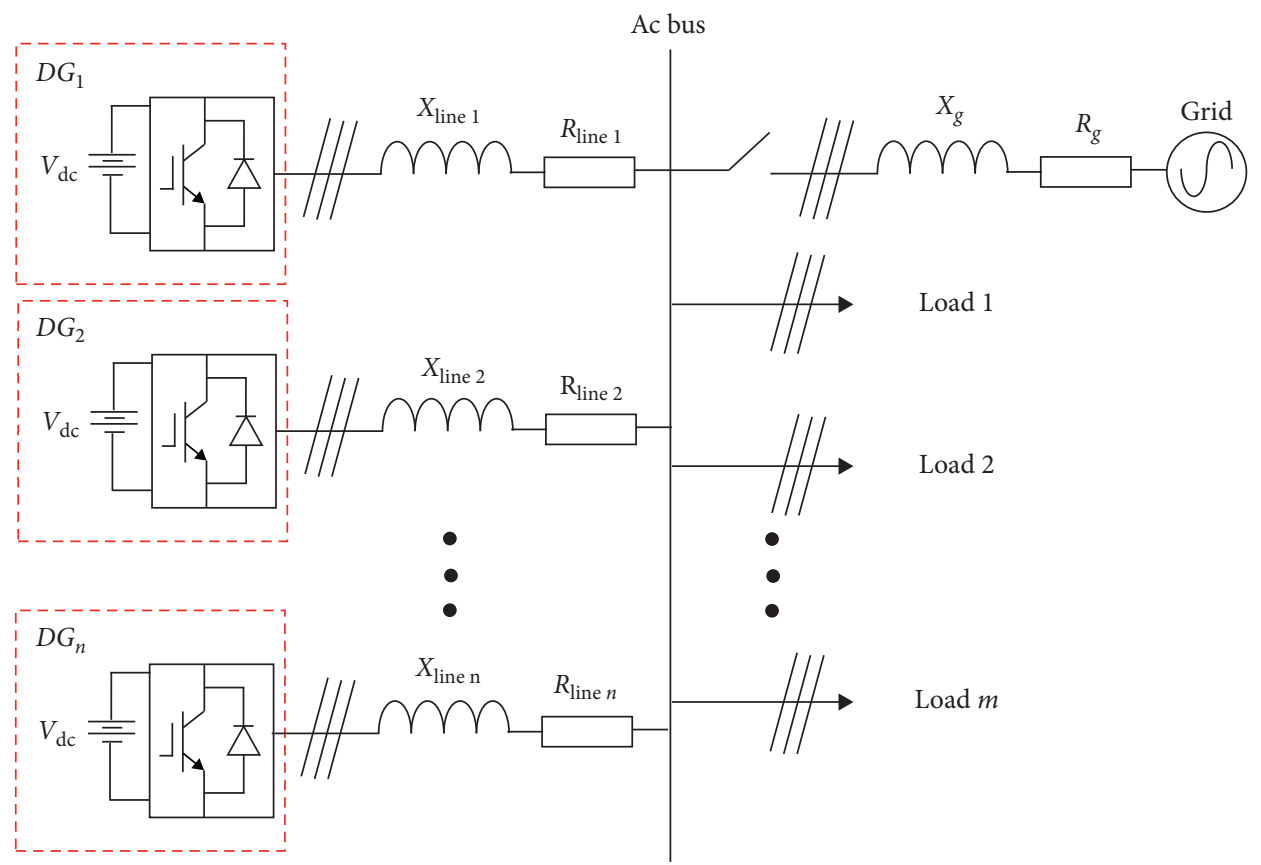

FIgURE 1: Typical microgrid-based VSI configuration [23].

MG operation. Thus, it includes the virtual impedance to balance lines impedance. The arctan droop control method used in the proposed control technique eliminates the droop slope of constant frequency and replaces it with an algorithm based on the arctan function. When the arctan-based power profile is utilized, the microgrid operating frequency is still within preset limits $[11,12]$. For its excellent transient performance, the FCS-MPC strategy is used to boost tracking performance and reduce the complexity compared to the traditional cascaded linear control method [9].

\section{The Existing Control Theory for VSI}

3.1. Arctan Droop Control. The droop functions may be linear or nonlinear. In any linear droop function scenario, droop function blocks may be explicitly substituted by unit gains. The resulting performance range scaling factor has no impact on system dynamics and steady state for linear droop function. The output range of linear droop lies in the range minus infinity to plus infinity. Therefore, any disturbance in the system can lead to large frequency deviations from the nominal values if determined by the same droop control method. An arctan-based droop control mechanism is introduced to prevent this specific issue. With the arctan frequency droop function, the frequency variance spectrum may be controlled within the preset values. The frequency variance spectrum may be further extended or minimized by specifying the output range scaling factor, while the voltage droop feature is always considered linear [21]. A comparison between arctan and the fixed gradient droop control strategy is shown in Figure 2. Li and Kao [24] highlighted that the conventional way to enhance the proportionate power sharing between the VSIs connected in parallel was to boost the droop gradient significantly. The introduction of the arctan function in the droop control method increases the tangential gradient around the power setpoint; therefore, a faster response can be obtained [11]. The arctan function enables voltage and frequency control to be achieved through variation of both the gradient and concavity [10]. The presumption that the arctan droop at frequency limits would have a slower response is the drawback of the arctan droop-based control methods. However, as high-level controls are generally used to change the power operating point dynamically, the frequency constraints' slower reaction is not a big contention point. It can be advantageous as it reduces the power to go out of limits [11].

Patel et al. [25] highlighted the shortcomings of the traditional droop control approach. An updated virtual droop control approach using the arctan feature for frequency control had been implemented to reduce the impact of impedance mismatch, and the response time has been improved.

Figure 3 shows the control strategy developed by [25].

In Figure 3, $V_{i}$ and $I_{o}$ are the output voltage and output current for the inverter. $\omega_{c}$ represents the low-pass filter cutoff frequency, $m$ and $n$ are the droop coefficients, $P$ is the active output power, $Q$ is the reactive output power, $P_{o}$ is the active reference power, $Q_{o}$ denotes the reactive reference power, and $f_{o}$ and $V_{o}$ denote the reference frequency and voltage, respectively.

Virtual impedance causes a "voltage drop" without impacting the physical loss of exchanged active/reactive power. This method improves the stability of the system and reduces circulating current among the microsources [26]. The implementation of such an approach is shown in the following equation.

$$
V_{\text {ref }}=V_{\text {droop }}-Z_{V(s)} * I_{o},
$$




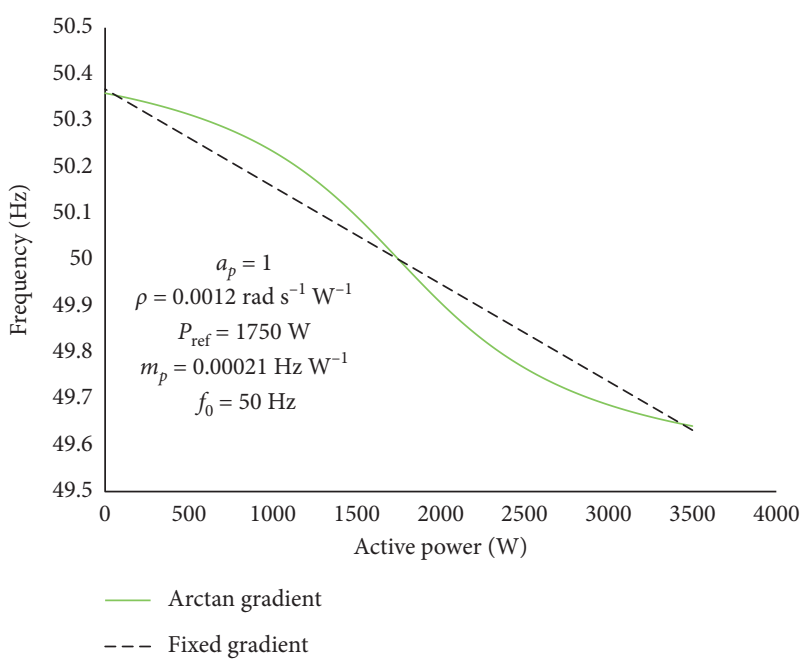

FIGURE 2: Comparison of arctan and fixed gradient droop control strategy.

where $V_{\text {droop }}$ denotes the reference voltage provided by the droop method, and $Z_{V(S)}$ is the virtual output impedance.

Guerero et al. [27] have shown how the calculation and design of virtual impedances are performed. In [10], the arctan droop control-based algorithm was used to overcome traditional droop control limitations, which always uses the constant frequency droop to control the frequency within predetermined limits. The control method showed better control concerning voltage and frequency in MG. It has demonstrated better control of the power profile's concavity and gradient than traditional power frequency droop with zero's constant concavity. The arctan-based algorithm is a monotonous rise in the magnitude of the preset boundary. The method was chosen because it showed a low complexity and required low computing time. The concavity is controlled by $\rho[10]$, and the bounds are governed by the parameter $a_{p}$, for example, Rowe et al. [11] have used $a_{p}$ equal to 1 , so as to make the frequency bounds to remain in the interval range of $[50-0.5,50+0.5]$. In general, the frequency can vary in the range of $\left[f_{o}-\left(a_{p} / 2\right), f_{o}+\left(a_{p} / 2\right)\right]$.

\subsection{Finite Control Set Model Predictive Control (FSC-MPC)} Control Method. An FCS-MPC approach depends on a mathematical system model to assess the impact of control measures on system response. Therefore, the action expected to minimize a specific objective function is implemented, and the process is repeated sequentially. Appropriate converters and filter models are needed to achieve good control efficiency when using this technique to VSI. The VSI threephase shown in Figure 4 is frequently used in AC microgrids converter topology. It can be seen that the output of VSI is attached to an LC filter that minimizes the switching harmonics.

The output voltage of VSI before passing the filter, which here is $V_{t}$, is calculated using the following equation:

$$
V_{t}=\frac{2}{3} V_{\mathrm{dc}} e^{i(j-1)(\pi / 3)}, \quad(j=0, \ldots, 7),
$$

where $V_{\mathrm{dc}}$ denotes the direct current (DC), and $V_{t}$ denotes the output voltage of the VSI.

By enumerating all potential control behaviors, FCSMPC anticipates the future conduct of the state component. The dynamic model of the system is thus required. Using Kirchhoff's laws, we can model the LC filter as follows [28]:

$$
\frac{\mathrm{d} x}{\mathrm{~d} t}=A x+B_{1} V_{t}+B_{2} i_{o}
$$

While

$$
\begin{aligned}
x & =\left[\begin{array}{c}
i_{f} \\
v_{p c}
\end{array}\right], \\
A & =\left[\begin{array}{cc}
\frac{-R_{f}}{L_{f}} & \frac{-1}{L_{f}} \\
\frac{1}{C_{f}} & 0
\end{array}\right], \\
B_{1} & =\left[\begin{array}{c}
\frac{1}{L_{f}} \\
0
\end{array}\right], \\
B_{2} & =\left[\begin{array}{c}
0 \\
-1 \\
C_{f}
\end{array}\right], \\
v_{p c} & =v_{c},
\end{aligned}
$$

equation (2) is used to calculate $V_{t}$; whereas, the measured variables are $i_{f}$ and $v_{c}$.

Depending on the sampling time $\left(T_{s}\right)$ method, the VSC discrete-time model can be obtained from equation (5) as follows:

$$
x(k+1)=A_{d} x(k)+B_{1 d} V_{t}(k)+B_{2 d} i_{o}(k) .
$$

While

$$
\begin{aligned}
A_{d} & =e^{A T_{s}}, \\
B_{1 d} & =\int_{0}^{T_{s}} e^{A \tau} B_{1} \mathrm{~d} \tau, \\
B_{2 d} & =\int_{0}^{T_{s}} e^{A \tau} B_{2} \mathrm{~d} \tau .
\end{aligned}
$$

The capacitor's predictive voltage is given by

$$
v_{P C}(k+1)=v_{P C}(k)+\frac{T_{s}}{C_{f}}\left(i_{f}(k)-i_{o}(k)\right) \text {. }
$$

The objective function can be represented in the traditional predictive voltage regulation using alpha-beta transformation as 


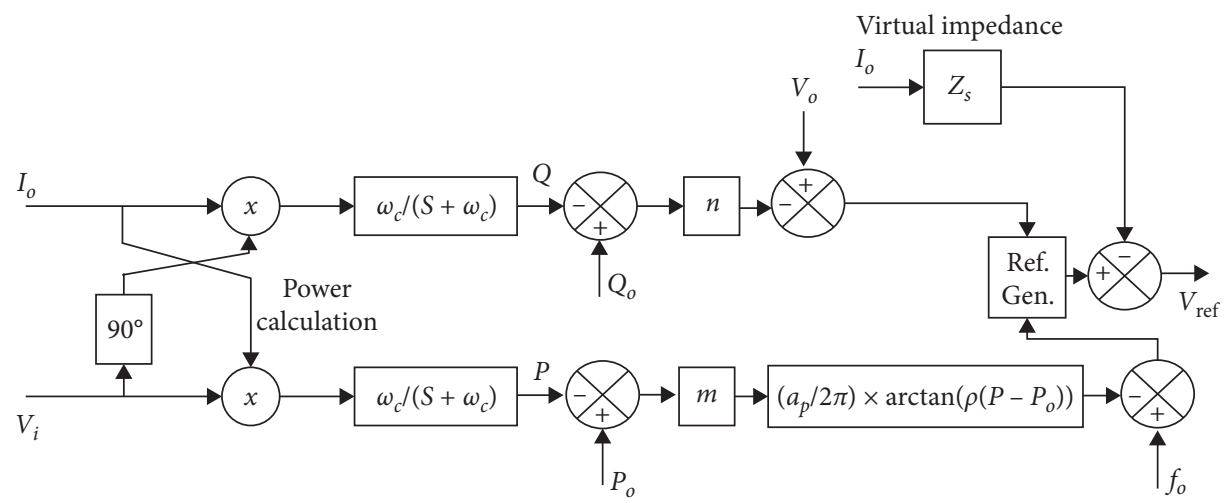

FIGURE 3: Arctan virtual impedance droop control strategy [25].

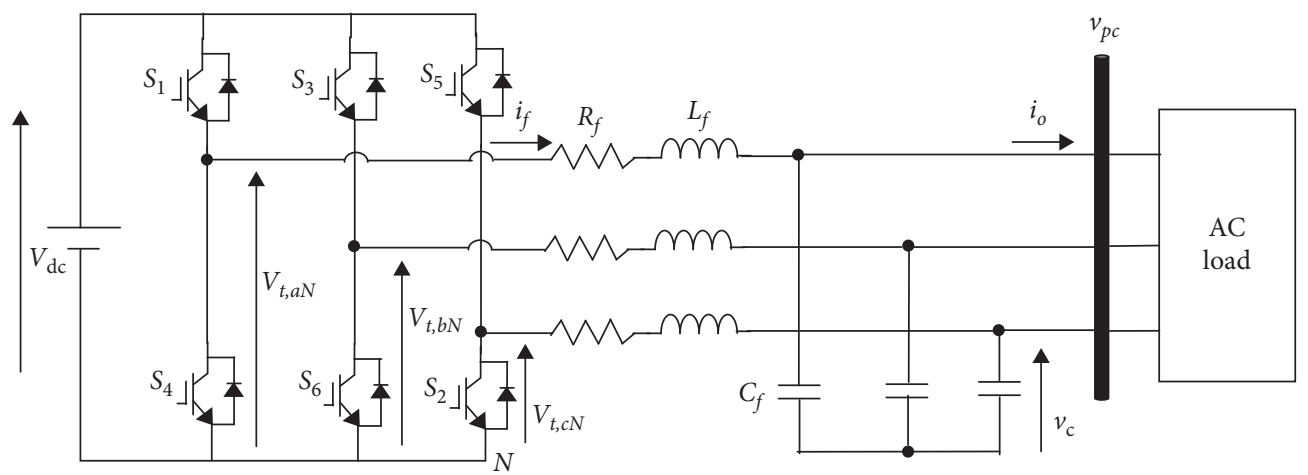

Figure 4: Typical three-phase VSI supplying a load in an MG.

$$
g_{p, i}=\left(v_{p c, \alpha}^{*}-v_{p c, \alpha}(k+1)\right)^{2}+\left(v_{p c, \beta}^{*}-v_{p c, \beta}(k+1)\right)^{2},
$$

where $v_{P C, \alpha}(k+1)$ and $v_{P C, \beta}(k+1)$ represent the real and imaginary parts of one-step prediction of the capacitor voltage, respectively. $v_{P C, \alpha}^{*}$ and $v_{P C, \beta}^{*}$ represent the real and imaginary parts of the reference voltage, respectively. Equation (8) shows how the minimization of error is obtained between the reference and the capacitor's predictive voltage.

3.3. Power Sharing Conditions in VSIs-Based Microgrids. The proportionate power sharing between the VSIs is obtained when the relationship of any two DGs' power and impedance in equation (9) is achieved.

$$
\begin{gathered}
\frac{P_{i}}{P_{j}}=\frac{Z_{j}}{Z_{i}}, \\
\frac{Q_{i}}{Q_{j}}=\frac{Z_{j}}{Z_{i}},
\end{gathered}
$$

equation (9) help us to write

$$
S_{1} Z_{1}=S_{2} Z_{2}=\cdots=S_{n} Z_{n}
$$

where $S=\sqrt{P^{2}+Q^{2}} . S$ denotes the apparent power; $Z_{i}$ and $Z_{j}$ represent output line impedances of different voltage source inverters.
In other words, this is generally satisfied when

$$
\begin{aligned}
& \frac{m_{1}}{m_{2}}=\frac{P_{2}}{P_{1}}=\cdots=\frac{m_{k}}{m_{k+1}}=\frac{P_{k+1}}{P_{k}}, \\
& \frac{n_{1}}{n_{2}}=\frac{Q_{2}}{Q_{1}}=\cdots=\frac{n_{k}}{n_{k+1}}=\frac{Q_{k+1}}{Q_{k}},
\end{aligned}
$$

$m, n$ are the active and reactive power droop coefficients, respectively. $P, Q$ are the active and reactive powers delivered by microsources in active and reactive power sharing with exact power ratio and droop coefficients.

Equation (11) is not satisfied when using the traditional droop control if the VSIs output impedances are not the same; thus, the introduction of fictitious impedance is required, so that the power sharing is proportionately assured [29].

\section{Proposed Control Algorithm}

The proposed control algorithm uses the arctan droop control with the virtual impedance strategy and the FCSMPC to gain the advantages of the two control strategies. Arctan droop control has demonstrated the ability to monitor the power profile's concavity and gradient compared to the conventional power frequency droop with a constant concavity of zero. As it is used with a virtual impedance control loop, it adds another advantage of monitoring the output power of different microsources 
interfaced with VSIs for different output impedances. Whereas, the FCS-MPC control strategy provides a benefit of hardware simplification as this replaces the voltage and current inner loops in the cascaded traditional droop control, robustness compared to conventional droop control, and track the reference voltage. Figure 5 illustrates the proposed control methodology in an MG of one VSI and a simple load.

In Figure 5, $\omega_{\text {nom }}$ is the nominal frequency, $V_{\text {nom }}$ is the nominal voltage, $P_{\text {ref }}$ is the reference active power, $Q_{\text {ref }}$ is the reference reactive power, $i_{f}$ is the inductor filter current, $v_{o}=v_{c}$ is the output voltage, $i_{o}$ is the output current, $v_{\text {ref }}$ is the output voltage reference droop with virtual impedance, and $v_{\text {droop }}$ denotes the droop voltage.

Figures 6-8 show the arctan droop control, virtual impedance control, and FSC-MPC control strategies diagrams, respectively.

The $P / \omega$ arctan droop can now be expressed as a nonlinear function:

$$
\omega=\omega_{\text {nom }}-2 a_{p} * \operatorname{Arctan}\left(\rho\left(P-P_{\text {ref }}\right)\right) .
$$

Or, it can also be expressed as

$$
f=f_{\text {nom }}-\frac{a_{p}}{\pi} * \operatorname{Arctan}\left(\rho\left(P-P_{\text {ref }}\right)\right),
$$

whereas the $Q / V$ droop is expressed as a linear function as follows:

$$
V_{\text {droop }}=V_{\text {nom }}-m_{q} *\left(Q-Q_{\text {ref }}\right) \text {, }
$$

where $a_{p}$ is the constant for controlling the bounds; in our study, it is chosen to be 1 to control the frequency in the bounds of $50 \pm 1 \% \mathrm{~Hz}, m_{q}$ is the droop coefficient for voltage control, and $\rho$ is a constant for controlling the concavity.

The reference voltage is given by

$$
v_{\text {ref }}=V_{\text {droop }}-i_{o} * z_{v} \text {. }
$$

The virtual impedance is given by

$$
\begin{aligned}
& z_{v}=R_{v} \mp \frac{\omega_{c} s L_{v}}{s+\omega_{c}}, \\
& v_{z v}=i_{o} * z_{v},
\end{aligned}
$$

where $L_{v}$ is a virtual inductance, $\omega_{c} /\left(s+\omega_{c}\right)$ represents the low pass filter, and $R_{v}$ represents the virtual resistance.

The one-step prediction for voltage using FSC-MPC is depicted in Figure 8, where the inputs are the output reference voltage after introducing the virtual impedance loop, the measured filter current, and the measured capacitor filter voltage.

\section{Results and Discussion}

All the simulations have been performed using Matlab/ Simulink software, and the proposed control strategy has been tested on the MG, which is composed of two microsources and linear loads $(R L)$, as shown in Figure 9.
In this study, the analyses include steady-state and load transient; the scenarios are as follows:

(a) Two VSIs with the same power outputs and identical output impedances

(b) Two VSIs with different power outputs and different output impedances

Two linear loads $(R L)$ have been considered for the study; the system parameters are indicated in Table 1.

In the normal operation mode, all parallel inverters and load 1 are always connected to PCC. At time $0.3 \mathrm{~s}$, load 2 is connected to PCC. A fault is introduced to the system during the time $0.5-0.501 \mathrm{~s}$. These scenarios are shown in Table 2 .

5.1. Two VSIs with the Same Power Outputs and Identical Output Impedances. The inverter active and reactive capacity should be DG1:DG2 $=1: 1$ ratio according to their droop coefficients, and the results showed that before $t=0.3 \mathrm{~s}$, the system was in steady-state operation, during that period, the voltage drops due to the rise of the load, but the voltage remained the same for all inverters. Their variations were in the range recommended by EN 50160 and IEEE1547 for low voltage networks; Figure 10 illustrates the variations for the two VSIs; as observed, the voltage decreased from 380.8 to $380.2 \mathrm{~V}$. On the side of frequency, the controller behaved as expected while keeping the frequency variation for both VSIs to remain in the set range, and the frequency decreased from $50 \mathrm{~Hz}$ to $49.75 \mathrm{~Hz}$ during the period $0-0.3 \mathrm{~s}$; this can be observed in Figure 11. The VSIs also delivered active and reactive power equitably as expected, and the results are presented in Figures 12-13. The results reveal that the inverters capacity ratio was $P 1: P 2=3 \mathrm{~kW}: 3 \mathrm{~kW}=m_{2}$ : $m_{1}=1: 1$ and $Q 1: Q 2=750 \mathrm{VAr}: 750 \mathrm{VAr}=n_{2}: n_{1}=1: 1$, which fulfills power sharing conditions.

At $t=0.3 \mathrm{~s}$, a load step change was introduced, and during the period $0.3-0.5 \mathrm{~s}$, the system remained stable while responding to the load added. The voltage and frequency for VSIs were decreased from $380.2 \mathrm{~V}$ to $380 \mathrm{~V}$ and from $49.75 \mathrm{~Hz}$ to $49.70 \mathrm{~Hz}$, respectively; this can be seen from Figures 10-11. The power sharing was also performed properly during this period, where the ratio was $P 1$ : $P 2=6 \mathrm{~kW}: 6 \mathrm{~kW}=m_{2}: m_{1}=1: 1$ and $Q 1: Q 2=1500 \mathrm{VAr}$ : $1500 \mathrm{VAr}=n_{2}: n_{1}=1: 1$ as shown in Figures 12-13.

At $0.5-0.501 \mathrm{~s}$, a fault was introduced to check the proposed controller's effectiveness after the occurrence of a transient during operation. According to Figures 10-13, the system remained stable after the fault has been cleared; during the fault, there was a reduction of active power and excessive reactive power demand.

At $0.501-0.8 \mathrm{~s}$, the voltage and frequency remained stable and the same as in the previous scenario. For power sharing, the VSIs continued to share the power as expected, and the ratio was $P 1: P 2=6 \mathrm{~kW}: 6 \mathrm{~kW}=Q 1: Q 2=1500 \mathrm{VAr}: 1500 \mathrm{VAr}=$ $m_{2}: m_{1}=n_{2}: n_{1}=1: 1$, as depicted on Figures 12-13.

During all scenarios, all the voltage and frequency variations were kept in the preset range values of $380 \mathrm{~V} \pm 5 \%$ and $50 \mathrm{~Hz} \pm 1 \%$, respectively; moreover, all the variations for 


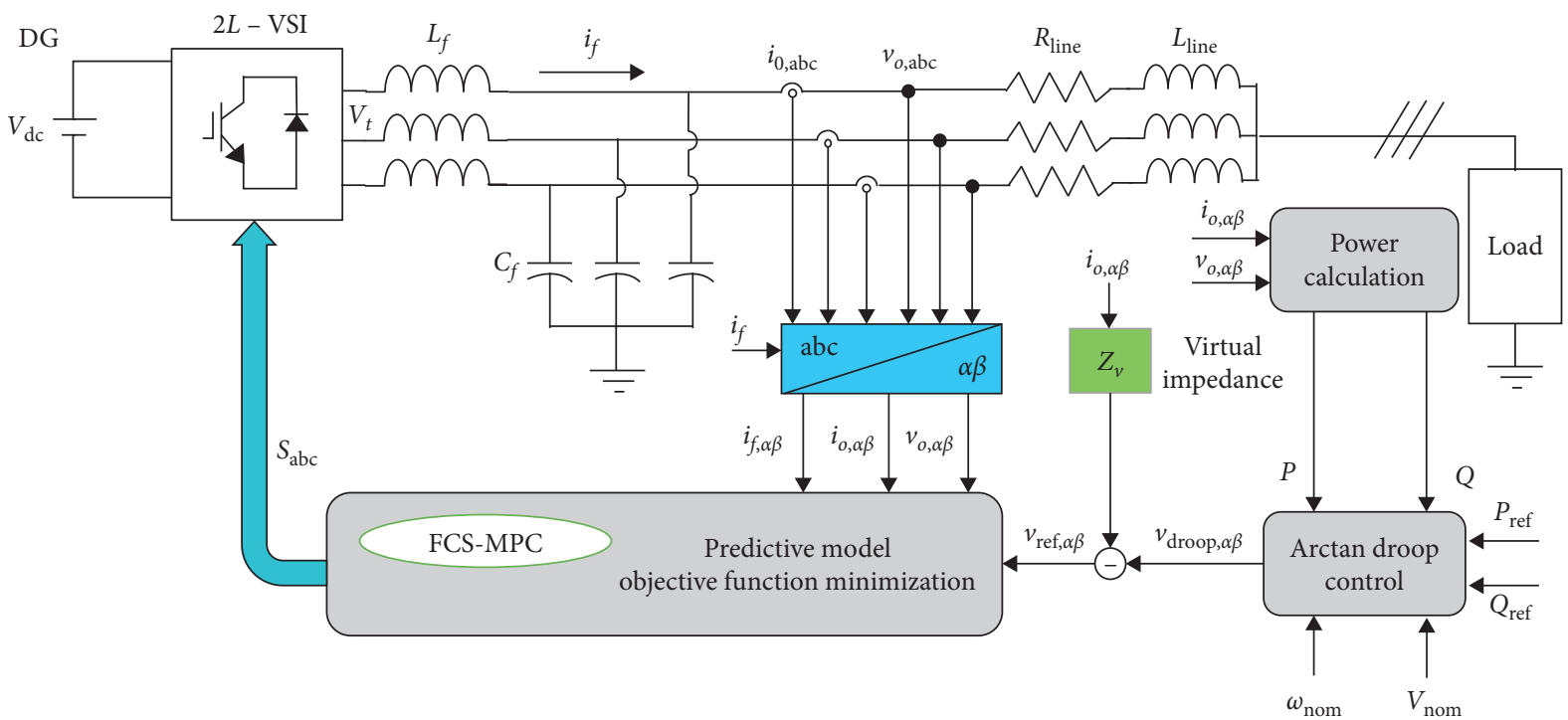

FIGURE 5: MPC-based arctan droop control strategy.

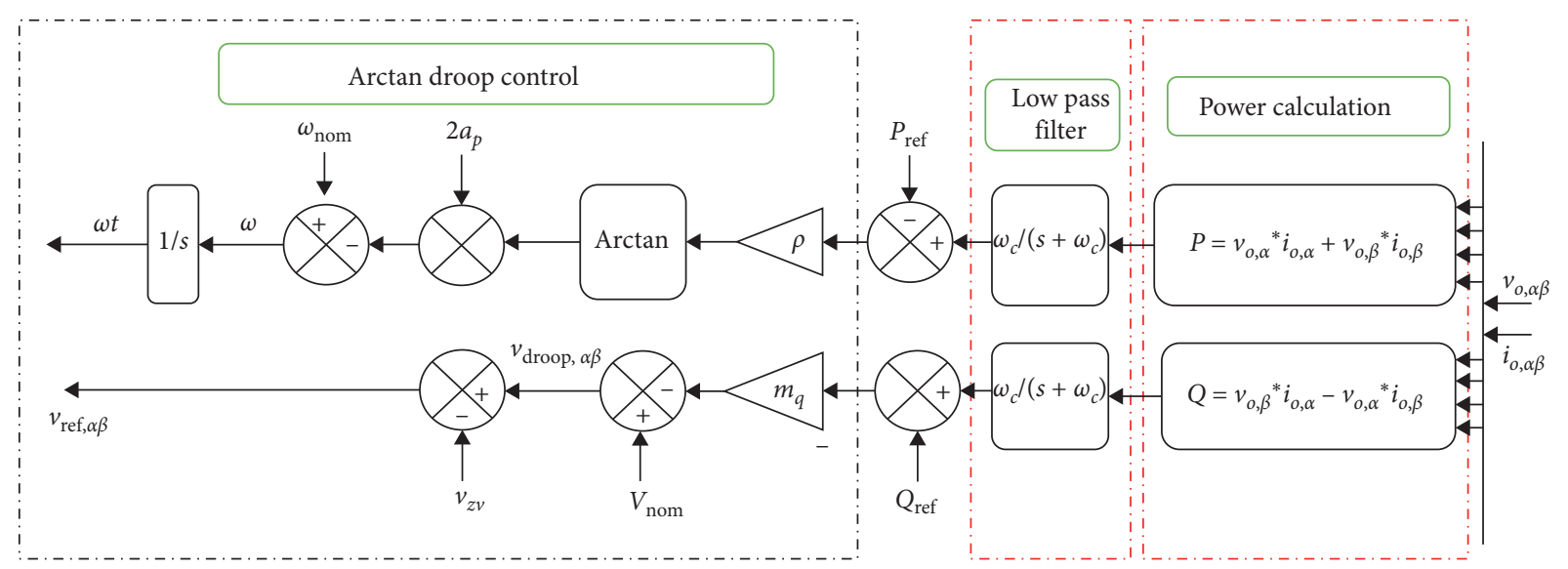

Figure 6: Arctan droop control diagram.

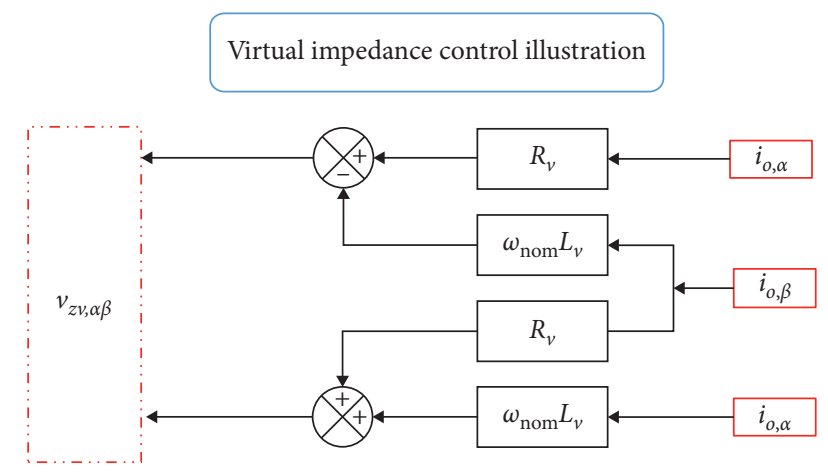

FIGURE 7: Virtual impedance control diagram.

voltages and frequency were kept in the range recommended by the IEEE 1547 standard. It has been noticed that there were no voltage and frequency deviations between inverters; hence, the proportional power sharing between the inverters is assured.
5.2. Two VSIs with Different Power Outputs and Different Output Impedances. The proposed strategy has also been tested on the parallel-connected VSIs with different powers and impedance outputs to study for its effectiveness. The parameters considered are given in Table 3.

The operation mode remains the same as in the previous case; however, the perfect power sharing ratio is $P 1$ : $P 2=m_{2}: m_{1}=2: 3$ and $Q 1: Q 2=n_{2}: n_{1}=1: 1$.

The proposed controller for VSIs connected in parallel is tested with two linear loads (RL). As highlighted in Figures 1415 , the proposed control strategy showed the ability to control the voltage and the frequency even during the load step change and faulty conditions. The voltage decreased during period $0-3 \mathrm{~s}$ for both DGs. It is stabilized at $V 1=V 2=380.2 \mathrm{~V}$ based on the voltage droop characteristics; when the VSIs supplied a permanent load, the frequency for both inverters decreased from $50 \mathrm{~Hz}$ to $49.75 \mathrm{~Hz}$. From $t=0.3-0.5 \mathrm{~s}$, load 2 was connected and the voltage decreased again to $V 1=V 2=379.8 \mathrm{~V}$, and the frequency was $f 1=f 2=49.70 \mathrm{~Hz}$. 


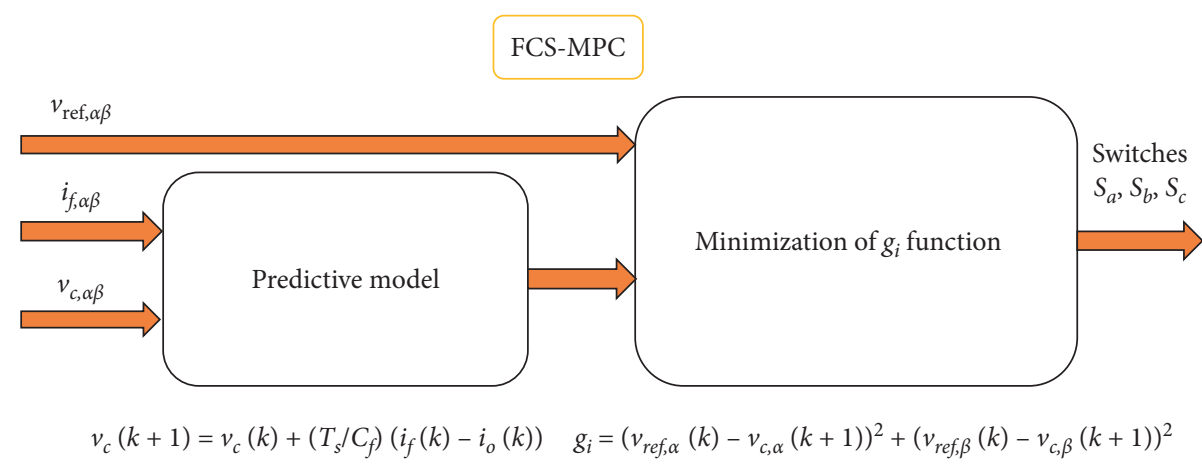

Figure 8: FSC-MPC control diagram.

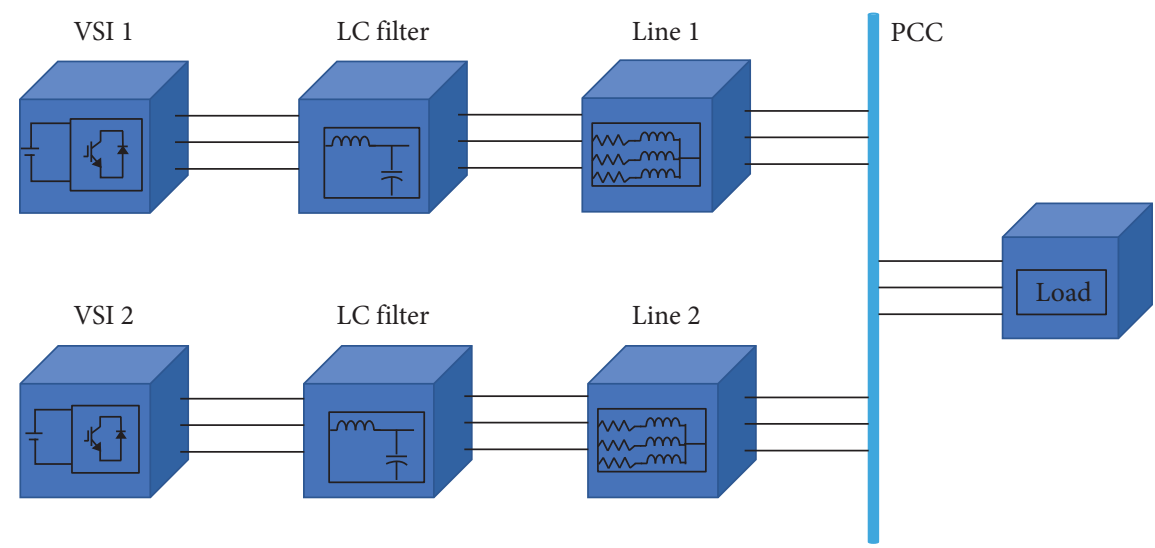

FIGURE 9: Microgrid network with parallel-connected VSIs.

TABle 1: System parameters.

\begin{tabular}{lc}
\hline Parameter & Value \\
\hline DC bus voltage & $700 \mathrm{~V}$ \\
Nominal bus frequency & $50 \mathrm{~Hz}$ \\
Sampling time & $T_{s}=30 \mathrm{us}$ \\
Nominal voltage & $380 \mathrm{~V}$ \\
Line impedance, DG1 & $0.528 \mathrm{mH}, 1.284 \Omega$ \\
Line impedance, DG2 & $0.528 \mathrm{mH}, 1.284 \Omega$ \\
Load 1 & $6000 \mathrm{~W}, 1500 \mathrm{VAr}$ \\
Load 2 & $6000 \mathrm{~W}, 1500 \mathrm{VAr}$ \\
Droop coefficients, DG1 & $3 e-5 \mathrm{~V} / \mathrm{W}, n_{1}=4 e-5 \mathrm{rad} / \mathrm{sVAr}$ \\
Droop coefficients, DG2 & $m_{1}=3 e-5 \mathrm{~V} / \mathrm{W}, n_{2}=4 e-5 \mathrm{rad} / \mathrm{sVAr}$ \\
The allowable frequency range of variation & $50 \mathrm{~Hz} \pm 1 \%$ \\
The allowable voltage range of variation & $m_{2}=380 \mathrm{~V} \pm 5 \%$ \\
\hline
\end{tabular}

TABle 2: Mode of operation.

\begin{tabular}{lcc}
\hline Event & Time & Operation \\
\hline Load step change & $0.3 \mathrm{~s}$, the breaker is on & All the two loads are connected \\
Fault & $0.5-0.501 \mathrm{~s}$ & The three-phase to ground fault was introduced to the system \\
\hline
\end{tabular}




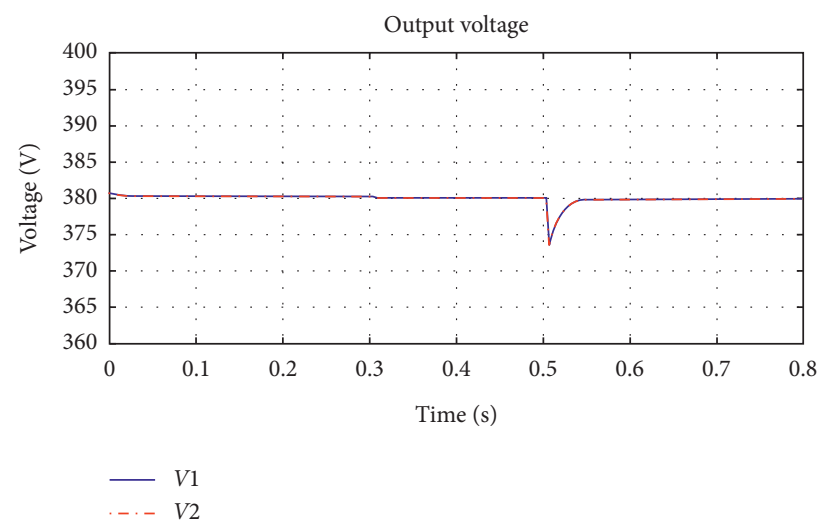

FIGURE 10: Voltage delivered by VSIs.

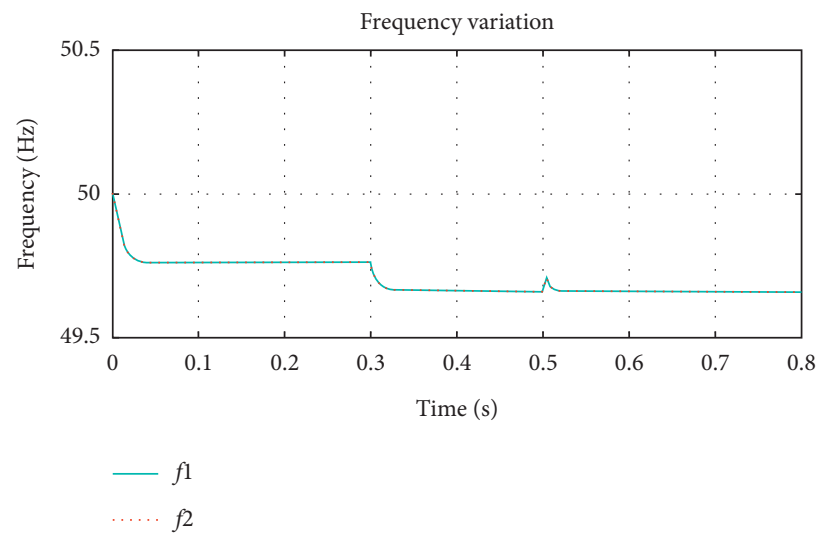

FIGURE 11: Frequency variation for VSI.

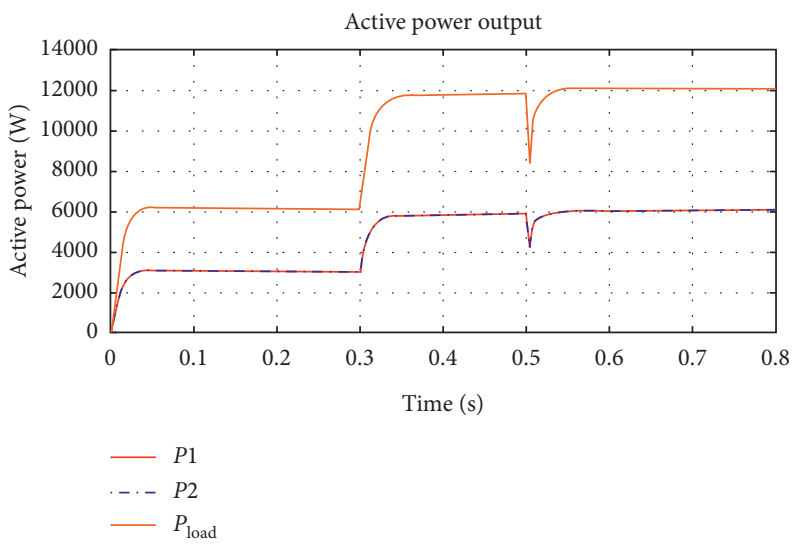

Figure 12: Active power sharing of VSIs.

The three-phase fault was introduced from time $0.5 \mathrm{~s}$ to $0.501 \mathrm{~s}$; during this period, there was a reduction of active power and excessive reactive power demand; however, the voltage and frequency variation range was $379.5-380.9 \mathrm{~V}$ and $49.70-49.73 \mathrm{~Hz}$, respectively. From 0.501 to 0.8 , the system remained stable and kept in the same conditions as the previous scenario. The voltages and frequency were kept within the allowable range of variations throughout the operation, as shown in Figures 14-
15. It has been noticed that the proposed controller continued to minimize deviations for voltage and frequency between the inverters.

Power sharing of parallel inverters was examined with a common load of $P_{\text {load }}=6000 \mathrm{~W}$ and $Q_{\text {load }}=1500 \mathrm{VAR}$, and at $t=0.3 \mathrm{~s}$, the sudden local load value of $P_{\text {load }}=6000 \mathrm{~W}$ and $Q_{\text {load }}=1500 \mathrm{VAR}$ is added. Two different line impedances were considered in this study. The MPC-based arctan droop control showed its capability to reduce the effect of line 


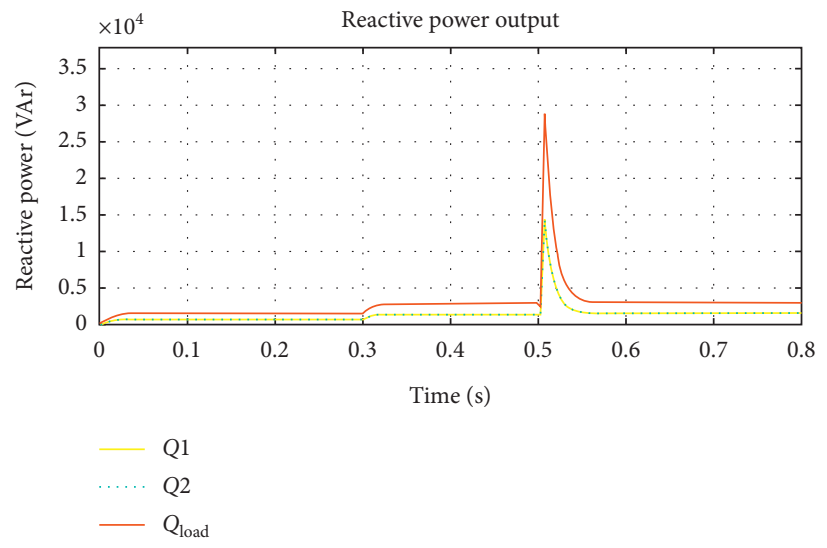

FIGURE 13: Reactive power sharing of VSIs.

TABLE 3: Tested data for different VSI's power outputs and impedances.

\begin{tabular}{lc}
\hline Parameter & Value \\
\hline DC bus voltage & $700 \mathrm{~V}$ \\
Nominal bus frequency & $50 \mathrm{~Hz}$ \\
Nominal voltage & $380 \mathrm{~V}$ \\
Sampling time & $T_{s}=30 \mathrm{us}$ \\
Line impedance, DG1 & $0.528 \mathrm{mH}, 1.284 \Omega$ \\
Line impedance, DG2 & $0.352 \mathrm{mH}, 0.856 \Omega$ \\
Load 1 & $6000 \mathrm{~W}, 1500 \mathrm{VAr}$ \\
Load 2 & $6000 \mathrm{~W}, 1500 \mathrm{VAr}$ \\
Droop coefficients, DG1 & $3 e-5 \mathrm{~V} / \mathrm{W}, n_{1}=4 e-5 \mathrm{rad} / \mathrm{sVAr}$ \\
Droop coefficients, DG2 & $\rho_{1} e-5 \mathrm{~V} / \mathrm{W}, n_{2}=4 e-5 \mathrm{rad} / \mathrm{sVAr}$ \\
& Virtual resistance of DG1 $=0 \Omega$ \\
Virtual impedance & Virtual resistance of DG2 $=0 \Omega$ \\
& The virtual inductance of DG1 $=1 \mathrm{mH}$ \\
The allowable frequency range of variation & The virtual inductance of DG2 $=1.176 \mathrm{mH}$ \\
The allowable voltage range of variation & $50 \mathrm{~Hz} \pm 1 \%$ \\
\hline
\end{tabular}

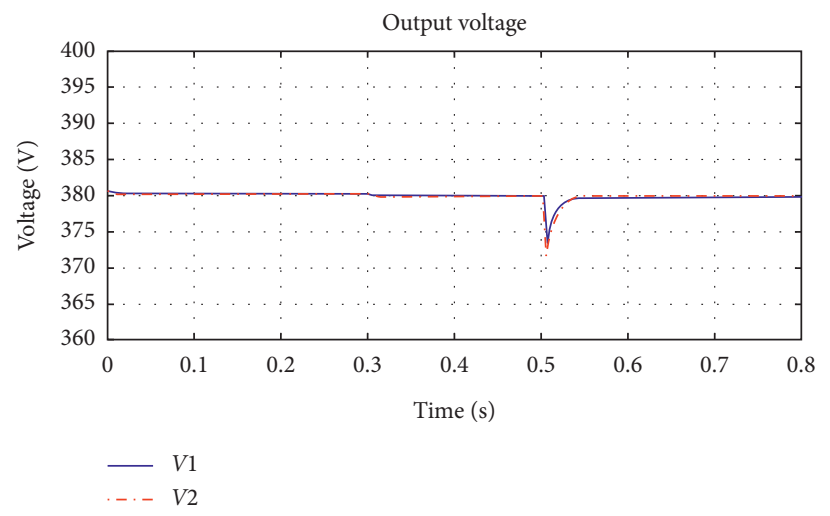

FIGURE 14: VSIs voltage outputs.

impedance difference on the parallel inverters to ensure power sharing among the inverters. The virtual inductor impedance was used to reduce the line impedance mismatch among the inverters, enhancing power decoupling and realizing the equal distribution of loads. From time $0-0.3 \mathrm{~s}$, $P 1=2500 \mathrm{~W}, \quad P 2=3800 \mathrm{~W}, \quad$ and $Q 1=748 \mathrm{kVAr}$,
Q2 $=752 \mathrm{kVAr}$, and when there was load change at $t=0.3-0.5 \mathrm{~s}$, the load 2 was connected to the system, and the power shared is as follows: $P 1=4800 \mathrm{~W}, P 2=7200 \mathrm{~W}$ and $Q 1=1500 \mathrm{VAr}, Q 2=1500 \mathrm{VAR}$; the same results hold after the fault was cleared; the power sharing results are highlighted in Figures 16-17. According to the results, the power 


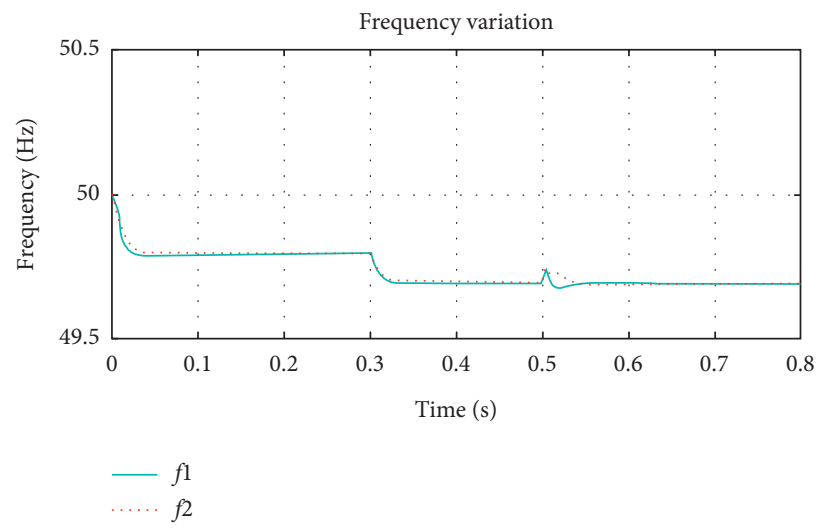

FIgURE 15: Frequency variation for VSIs.

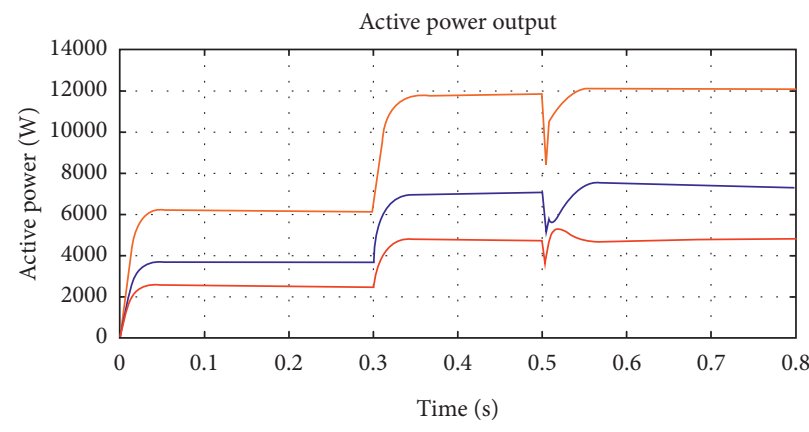

$-P 1$

$-P 2$

$P_{\text {load }}$

FIgURE 16: Active power sharing for VSIs.

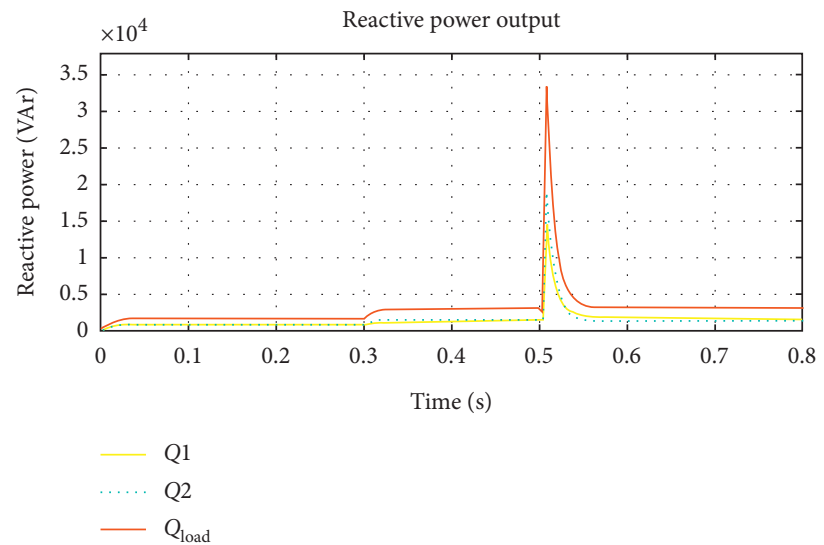

FIGURE 17: Reactive power sharing for VSIs.

sharing ratio in all scenarios is as follows: $P 1: P 2=m_{2}$ : $m_{1}=2: 3$ and $Q 1: Q 2=m_{2}: m_{1}=n_{2}: n_{1}=1: 1$, which is following the condition for good power sharing in this study.

The findings showed the voltage THD evaluation of $1.23 \%$ for the studied system, which is below the one recommended by EN 61000-2-2 and IEC62040-3 in low voltage networks; Figure 18 shows the voltage THD evaluation. The designed control strategy shows its superiority in voltage THD reduction compared to the other controllers, presented in Table 4. 


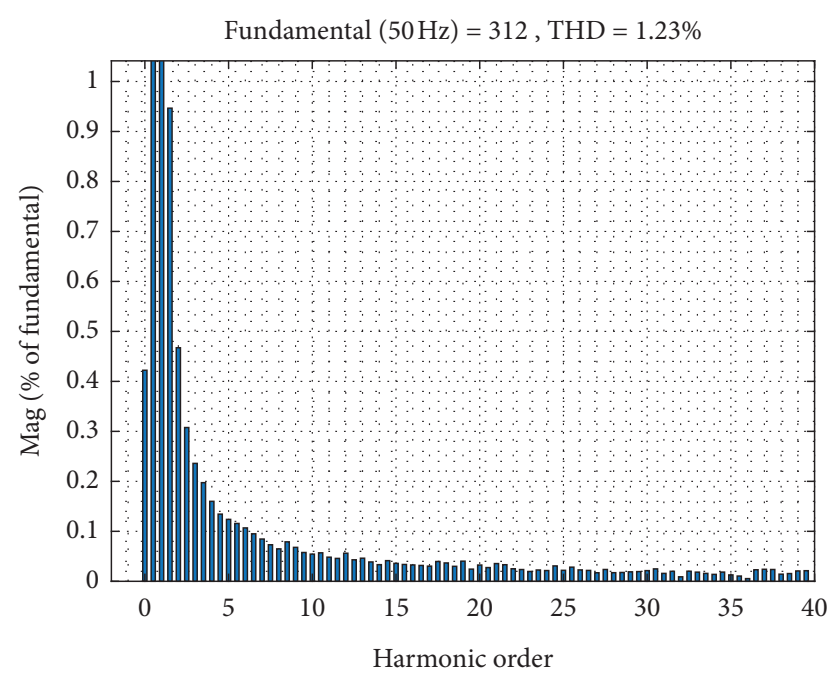

Figure 18: Voltage THD evaluation.

TABLE 4: Comparison of different control strategies based on voltage THD.

\begin{tabular}{lcc}
\hline Reference & Control strategy & Voltage THD (\%) \\
\hline$[30]$ & FSC-MPC & 2.05 \\
{$[31]$} & FSC-MPC with improved droop control & 3.8 \\
{$[32]$} & Deadbeat control & 2.8 \\
{$[33]$} & Modulated MPC & 2.54 \\
{$[9]$} & FSC-MPC with a current observer & 2.71 \\
Proposed control strategy & MPC-based arctan droop control & 1.23 \\
\hline
\end{tabular}

\section{Conclusion}

This study proposes a hybrid control method called MPCbased arctan droop control to control the parallel-connected VSIs in an islanded microgrid. The voltage and frequency were adequately monitored. The proposed control strategy has been tested in an MG with VSIs of the same power ratings and same output impedance and VSIs of different power ratings and different output impedances. The proposed control strategy demonstrated promising results in controlling the MG frequency and voltage according to EN 50160 and IEEE 1547-2018. It also showed the ability to control the VSIs to share power equitably in a microgrid. Various simulation results demonstrate that the proposed control method is feasible and effective.

\section{Data Availability}

The data used to support the findings of this study are included within the article.

\section{Conflicts of Interest}

The authors declare that they have no conflicts of interest.

\section{Acknowledgments}

This research was supported by the Pan African University, Institute for Basic Sciences, Technology and Innovation, in the form of postgraduate student research funding.

\section{References}

[1] C. Wang, X. Yang, Z. Wu et al., "A highly integrated and reconfigurable microgrid testbed with hybrid distributed energy sources," IEEE Transactions on Smart Grid, vol. 7, no. 1, pp. 451-459, 2016.

[2] R. H. Lasseter, "Microgrids and distributed generation," Journal of Energy Engineering, vol. 133, no. 3, pp. 144-149, 2007.

[3] M. Alhasheem, T. Dragicevic, F. Blaabjerg, and P. Davari, "Parallel operation of dual VSCs regulated by FCS-MPC using droop control approach," in Proceedings of the European Conference on Power Electronics and Applications, Riga, Latvia, 2018.

[4] U. B. Tayab, M. A. B. Roslan, L. J. Hwai, and M. Kashif, "A review of droop control techniques for microgrid," Renewable and Sustainable Energy Reviews, vol. 76, pp. 717-727, 2017.

[5] M. A. Hossain, H. R. Pota, W. Issa, and M. J. Hossain, "Overview of AC microgrid controls with inverter-interfaced generations," Energies, vol. 10, no. 9, pp. 1-27, 2017.

[6] K. De Brabandere, B. Bolsens, J. Van Den Keybus, A. Woyte, J. Driesen, and R. Belmans, "A voltage and frequency droop control method for parallel inverters," IEEE Transactions on Power Electronics, vol. 22, pp. 1107-115, 2007.

[7] A. J. Babqi and A. H. Etemadi, "MPC-based microgrid control with supplementary fault current limitation and smooth transition mechanisms," IET Generation, Transmission \& Distribution, vol. 11, no. 9, pp. 2164-2172, 2017.

[8] T. Dragicevic, "Model predictive control of power converters for robust and fast operation of AC microgrids," IEEE Transactions on Power Electronics, vol. 33, no. 7, pp. 63046317, 2018. 
[9] T. Chen, O. Abdel-Rahim, F. Peng, and H. Wang, "An improved finite control set-MPC-based power sharing control strategy for islanded AC microgrids," IEEE Access, vol. 8, pp. 52676-52686, 2020.

[10] A. E. M. Bouzid, P. Sicard, H. Chaoui, A. Cheriti, M. Sechilariu, and J. M. Guerrero, "A novel decoupled trigonometric saturated droop controller for power sharing in islanded low-voltage microgrids," Electric Power Systems Research, vol. 168, no. 7, pp. 146-161, 2019.

[11] C. N. Rowe, T. J. Summers, R. E. Betz, D. J. Cornforth, and T. G. Moore, "Arctan power-frequency droop for improved microgrid stability," IEEE Transactions on Power Electronics, vol. 28, no. 8, pp. 3747-3759, 2013.

[12] C. N. Rowe, T. J. Summers, R. E. Betz, C. N. Rowe, T. J. Summers, and R. E. Betz, "Arctan power frequency droop for power electronics dominated microgrids arctan power frequency droop for power electronics dominated microgrids," in Proceedings of the 2010 20th Australasian Universities Power Engineering Conference, vol. 10, no. 2, pp. 157-165, Christchurch, New Zealand, 2013.

[13] H. Yu, P. Zeng, Z. Li, C. Zang, and S. Li, "Fully distributed hierarchical control of parallel grid-supporting inverters in islanded AC microgrids," IEEE Transactions on Industrial Informatics, vol. 14, no. 2, pp. 679-690, 2017.

[14] P. Cortés, M. P. Kazmierkowski, R. M. Kennel, D. E. Quevedo, and J. Rodriguez, "Predictive control in power electronics and drives," IEEE Transactions on Industrial Electronics, vol. 55, no. 12, pp. 4312-4324, 2008.

[15] T. John, S. Member, Y. Wang, S. Member, K. T. Tan, and P. L. So, "Coordinated operation of a microgrid with a distribution network device," in Proceedings of the IEEE Innovative Smart Grid Technologies-Asia, pp. 1-6, ISGT ASIA, Bangkok, Thailand, 2015.

[16] P. Cortés, G. Ortiz, J. I. Yuz, J. Rodriguez, S. Vazquez, and L. G. Franquelo, "Model predictive control of an inverter with output LC filter for UPS applications," IEEE Transactions on Industrial Electronics, vol. 56, no. 6, pp. 1875-1883, 2009.

[17] C. Zheng, T. Dragicevic, and F. Blaabjerg, "Current-sensorless finite-set model predictive control for LC-filtered voltage source inverters," IEEE Transactions on Power Electronics, vol. 35, no. 1, pp. 1086-1095, 2019.

[18] C. Zheng, T. Dragičević, B. Majmunović, and F. Blaabjerg, "Constrained modulated model-predictive control of an LCfiltered voltage-source converter," IEEE Transactions on Power Electronics, vol. 35, no. 2, pp. 1967-1977, 2020.

[19] H. A. Young, M. P. Kazmierkowski, J. R. Espinoza et al., "State of the art of finite control set model predictive control in power electronics," IEEE Transactions on Industrial Informatics, vol. 9, no. 2, pp. 1003-1016, 2012.

[20] J. Hu, Y. Shan, J. M. Guerrero, A. Ioinovici, K. W. Chan, and J. Rodriguez, "Model predictive control of microgrids-an overview," Renewable and Sustainable Energy Reviews, vol. 136, 2021.

[21] K. Manjunath and V. Sarkar, "Performance assessment of different droop control techniques in an AC microgrid," in Proceedings of the 2017 7th International Conference on Power Systems, pp. 93-98, ICPS, Pune, India, 2017.

[22] Y. Shan, J. Hu, Z. Li, and J. M. Guerrero, "A model predictive control for renewable energy based AC microgrids without any PID regulators," IEEE Transactions on Power Electronics, vol. 33, no. 11, pp. 9122-9126, 2018.

[23] L. Ruiming and W. Shengtie, "Power distribution of parallel converters in islanded microgrid using virtual resistance droop control," in Proceedings of the 2018 13th IEEE
Conference on Industrial Electronics and Applications (ICIEA), pp. 1671-1675, Wuhan, China, 2018.

[24] Y. W. Li and C. N. Kao, "An accurate power control strategy for power-electronics-interfaced distributed generation units operating in a low-voltage multibus microgrid," IEEE Transactions on Power Electronics, vol. 24, no. 12, pp. 29772988, 2009.

[25] U. N. Patel, D. Gondalia, and H. H. Patel, "Modified droop control scheme for load sharing amongst inverters in a micro grid," Advances in Energy Research, vol. 3, no. 2, pp. 81-95, 2015.

[26] J. M. Guerrero, C. Raj, and D. N. Gaonkar, "Power-sharing control strategy of parallel inverters in AC microgrid using improved reverse droop control," International Journal of Power Electronics, vol. 11, no. 1, pp. 116-137, 2020.

[27] J. M. Guerrero, L. G. De Vicuña, J. Matas, J. Miret, and M. Castilla, "Output impedance design of parallel-connected UPS inverters," IEEE Transactions on Industrial Electronics, vol. 2, no. 4, pp. 1123-1128, 2004.

[28] H. J. Yoo, T. T. Nguyen, and H. M. Kim, "MPC with constant switching frequency for inverter-based distributed generations in microgrid using gradient descent," Energies, vol. 12, no. 6, pp. 7-9, 2019.

[29] J. Kim, J. M. Guerrero, P. Rodriguez, R. Teodorescu, and K. Nam, "Mode adaptive droop control with virtual output impedances for an inverter-based flexible AC microgrid," IEEE Transactions on Power Electronics, vol. 26, no. 3, pp. 689-701, 2011.

[30] V. Yaramasu, M. Rivera, M. Narimani, B. Wu, and J. Rodriguez, "Model predictive approach for a simple and effective load voltage control of four-leg inverter with an output LC filter," IEEE Transactions on Industrial Electronics, vol. 61, no. 10, pp. 5259-5270, 2014.

[31] Y. Li, Z. Zhang, S. Alireza Davari, C. Garcia, and J. Rodriguez, "FCS-MPC based primary control with improved performance for islanded AC microgrids," in Proceedings of the 2020 11th Power Electronics, Drive Systems, and Technologies Conference (PEDSTC), Tehran, Iran, 2020.

[32] P. Mattavelli, "An improved deadbeat control for UPS using disturbance observers," IEEE Transactions on Industrial Electronics, vol. 52, no. 1, pp. 206-212, 2005.

[33] S. K. Gannamraju, D. Valluri, and R. Bhimasingu, "Comparison of fixed switching frequency based optimal switching vector MPC algorithms applied to voltage source inverter for stand-alone applications," in Proceedings of the 2019 National Power Electronics Conference (NPEC), Tiruchirappalli, India, 2019. 